\title{
Vector-valued nonuniform multiresolution analysis related to Walsh function
}

\author{
Abdullah * \\ College of Engineering, Qassim University, Buraidah 51452, Al-Qassim, KSA
}

\begin{abstract}
In this paper, we introduce vector-valued nonuniform multiresolution analysis on positive half-line related to Walsh functions. We obtain the necessary and sufficient condition for the existence of associated wavelets.
\end{abstract}

Keywords Vector-valued Wavelets, Multiresolution analysis, Scaling function, Wash-Fourier transform.

AMS 2010 subject classifications 42C15, 40A30

DOI: $10.19139 /$ soic-2310-5070-681

\section{Introduction}

The idea of multiresolution analysis has become one of the main tools for constructing wavelets. In his paper, Mallat [15] first formulated the remarkable idea of multiresolution analysis (MRA) that deals with a general formlism for construction of an orthogonal basis of wavelet bases. A multiresolution analysis consists of a sequence of embedded closed subspaces $\left\{V_{j}: j \in \mathbb{Z}\right\}$ for approximating $L^{2}(\mathbb{R})$ functions such that $\cap_{j \in \mathbb{Z}} V_{j}=\{0\}$, $\cup_{j \in \mathbb{Z}} V_{j}$ is dense in $L^{2}(\mathbb{R})$ and which satisfies $f(x) \in V_{j}$ if and only if $f(2 x) \in V_{j+1}$. Furthermore, there exist an element $\varphi \in V_{0}$ such that the collection of integer translates of $\varphi,\{\varphi(x-n)\}_{n \in \mathbb{Z}}$ is a complete orthonormal system for $V_{0}$. Any compactly supported wavelet must come from a MRA. The function $\varphi \in V_{0}$ is called a scaling function.

The idea of wavelet and multiresolution analysis has been extended to many different setups. One can replace the dilation factor 2 by an integer $N \geq 2$ and one needs to construct $N-1$ wavelets to generate the whole space $L^{2}(\mathbb{R})$. In general, in higher dimensions, it can be replaced by a dilation matrix $A$, in which case the number of wavelets required is $|\operatorname{det} A|-1$. But in all these cases, the translation set is always a group. In the two papers [7], [8], Gabardo and Nashed considered a generalization of Mallat's [15] celebrated theory of MRA, in which the translation set acting on the scaling function associated with the MRA to generate the subspace $V_{0}$ is no longer a group, but is the union of $\mathbb{Z}$ and a translate of $\mathbb{Z}$. More precisely, this set is of the form $\{0, r / N\}+2 \mathbb{Z}$, where $N \geq 1$ is an integer, $1 \leq r \leq 2 N-1, r$ is an odd integer relatively prime to $N$. They call this a nonuniform multiresolution analysis (NUMRA) and is based on the theory of spectral pairs.

The idea of dyadic multiresolution analysis has been studied on $L^{2}\left(\mathbb{R}^{+}\right)$, where $\mathbb{R}^{+}$denotes the positive halfreal line by Protasov and Farkov. Farkov [5] has given a general construction of compactly supported orthogonal p-wavelets in $L^{2}\left(\mathbb{R}^{+}\right)$. Farkov et al. [6] gave an algorithm for biorthogonal wavelets related to Walsh functions on positive half line. Protasov and Farkov constructed examples of refinable functions that generate MRA on halfline and Vilenkin groups with the help of Cohen conditions in [18]. F. A. Shah [19], studied the construction

\footnotetext{
${ }^{*}$ Correspondence to: Abdullah (E-mail: abdullahdu@qec.edu.sa; abd.zhc.du@gmail.com). College of Engineering, Qassim University,
} Buraidah 51452, Al-Qassim, KSA.

ISSN 2310-5070 (online) ISSN 2311-004X (print)

Copyright (C) 2020 International Academic Press 
of $p$-wavelet packets associated with the multiresolution analysis defined by Farkov [5], for $L^{2}\left(\mathbb{R}^{+}\right)$. Recently, Meenakshi et al. [17] studied NUMRA on positive half line and they also derived the analogue of Cohen's condition for the nonuniform multiresolution analysis on the positive half-line.

In [24], Xia and Suter, introduced vector-valued multiresolution analysis and vector-valued wavelets for vector-valued signal spaces. Sun and Cheng, introduced vector-valued multiresolution analysis with dilation factor $\alpha \geq 2$ and orthogonal vector-valued wavelets in [23]. The properties of vector-valued wavelets packets are presented in [3]. In [1], Abdullah, introduced vector-valued multiresolution analysis on local field of positive characteristic and obtained the necessary and sufficient condition for the existence of associated wavelets.

In the present paper, we study vector-valued nonuniform multiresolution analysis on positive half-line and we obtain the necessary and sufficient condition for the existence of associated wavelets. The paper is organized as follows. In Sec. 2, we discuss some preliminary facts about NUMRA on positive half-line. We introduce the notion of VNUMRA on positive half-line and find a necessary and sufficient condition for the existence of associated wavelets in Sec. 3.

\section{Nonuniform multiresolution analysis on positive half-line}

Let $\mathbb{Z}^{+}$and $\mathbb{R}^{+}$denote the set of positive integers and positive real numbers, respectively. For the basic results related to Walsh-Fourier analysis and definition of multiresolution $p$-analysis on $L^{2}\left(\mathbb{R}^{+}\right.$), we refer to ( [5], [6], [17], [19], [20], [21], [22] ).

Let $N$ be an integer, $N \geq 1$ and an odd integer $r$ with $1 \leq r \leq 2 N-1, r$ and $N$ are relatively prime, we consider the translation set $\Lambda_{r, N}^{+}$as

$$
\Lambda_{r, N}^{+}=\left\{0, \frac{r}{N}\right\}+\mathbb{Z}^{+}=\left\{\frac{r k}{N}+n: n \in \mathbb{Z}^{+}, k=0,1\right\} .
$$

Let $[x]$ denotes integer part of $x$. For $x \in \mathbb{R}^{+}$and for any positive integer $j$, we set

$$
x_{j}=\left[N^{j} x\right](\bmod N), x_{-j}=\left[N^{1-j} x\right](\bmod N),
$$

where $x_{j}, x_{-j} \in\{0,1, \ldots, N-1\}$.

Non-uniform multiresolution analysis in $L^{2}\left(\mathbb{R}^{+}\right)$defined by Meenakshi et. al. is as follows:

Definition 2.1. A nonuniform multiresolution analysis on positive half-line is a sequence of closed subspaces $V_{j} \subset L^{2}\left(\mathbb{R}^{+}\right), j \in \mathbb{Z}$ such that the following properties hold:

(1) $V_{j} \subset V_{j+1}$ for all $j \in \mathbb{Z}$,

(2) $\bigcup_{j \in \mathbb{Z}} V_{j}$ is dense in $L^{2}(\mathbb{R})$ and $\bigcap_{j \in \mathbb{Z}} V_{j}=\{0\}$,

(3) $f(.) \in V_{j}$ if and only if $f(N.) \in V_{j+1}$,

(4) There exists a function $\varphi$ in $V_{0}$ such that $\left\{\varphi(. \ominus \lambda): \lambda \in \Lambda_{r, N}^{+}\right\}$where $\Lambda_{r, N}^{+}=\{0, r / N\}+\mathbb{Z}^{+}$, is a complete orthonormal system for $V_{0}$.

The function $\varphi$ whose existence is asserted in (4) is called a scaling function of the given NUMRA.

When, $N>1$, the dilation factor of $N$ ensures that $N \Lambda_{r, N}^{+} \subset \mathbb{Z}^{+} \subset \Lambda_{r, N}^{+}$. Condition (3) and (4) implies that

$$
\varphi_{1, \lambda}(x)=N^{1 / 2} \varphi(N x \ominus \lambda), \quad \lambda \in \Lambda_{r, N}^{+}
$$


constitute and orthonormal basis in $V_{1}$.

Since $V_{0} \subset V_{1}$, the function $\varphi \in V_{1}$ and has the Fourier expansion

$$
\varphi(x)=\sum_{\lambda \in \Lambda^{+}} h_{\lambda}(N)^{1 / 2} \varphi(N x \ominus \lambda)
$$

where $h_{\lambda}=\int_{\mathbb{R}^{+}} \varphi(x) \overline{\varphi_{1, \lambda}(x)} d x$.

This implies that

$$
\varphi(x)=N \sum_{\lambda \in \Lambda^{+}} a_{\lambda} \varphi(N x \ominus \lambda), \quad \sum_{\lambda \in \Lambda^{+}}\left|a_{\lambda}\right|^{2}<\infty
$$

where $a_{\lambda}=N^{-1 / 2} h_{\lambda}$.

Now, we consider $W_{0}$ the orthogonal complement of $V_{0}$ on $V_{1}$, i.e.,

$$
V_{1}=V_{0} \oplus W_{0} .
$$

If $\psi_{1}, \psi_{2}, \ldots, \psi_{N-1}$ are the functions in $W_{0}$, then for $\ell=0,1, \ldots, N-1$, there exists sequences $\left\{a_{\lambda}^{\ell}\right\}_{\lambda \in \Lambda^{+}}$ satisfying $\sum_{\lambda \in \Lambda^{+}}\left|a_{\lambda}^{\ell}\right|^{2}<\infty$ such that

$$
\psi_{\ell}(x)=N \sum_{\lambda \in \Lambda^{+}} a_{\lambda}^{\ell} \varphi(N x \ominus \lambda)
$$

On taking the Walsh-Fourier transform, we have

$$
\hat{\psi}_{\ell}(\xi)=m_{\ell}\left(\frac{\xi}{N}\right) \hat{\varphi}\left(\frac{\xi}{N}\right)
$$

where the functions $m_{\ell}(\xi)=\sum_{\lambda \in \Lambda^{+}} a_{\lambda}^{\ell} \overline{\chi(\lambda, \xi)}$ and $\chi(\lambda, \xi)=\exp \left(\frac{2 \pi i}{N} \sum_{j=1}^{\infty}\left(\lambda_{j} \xi_{-j}+\lambda_{-j} \xi_{j}\right)\right)$ where $\lambda_{j}$ and $\xi_{j}$ are calculated by (2.1) for a positive integer $N$.

Since $\Lambda_{r, N}^{+}=\{0, r / N\}+\mathbb{Z}^{+}$, we can write that

$$
m_{\ell}(\xi)=m_{\ell}^{1}(\xi)+\overline{\chi\left(\frac{r}{N}, \xi\right)} m_{\ell}^{2}(\xi), \quad \ell=0,1, \ldots, N-1,
$$

where $m_{\ell}^{1}$ and $m_{\ell}^{2}$ are locally $L^{2}$ functions.

In this case $\left\{\psi_{1}, \psi_{2}, \ldots, \psi_{N-1}\right\}$ is a set of basic wavelets associated with a scaling function $\varphi$. It is easy to show that $\left\{\psi_{\ell}(x \ominus \lambda): 1 \leq \ell \leq N-1\right\}$ is an orthonormal basis of $W_{0}$. An obvious rescaling shows that

$$
\left\{\psi_{\ell, j, \lambda}=(N)^{j / 2} \psi_{\ell}\left((N)^{j} x \ominus \lambda\right): 1 \leq \ell \leq N-1, \lambda \in \Lambda_{r, N}^{+}\right\}
$$

is an orthonormal basis of $W_{j}$. Since $\cup_{j \in \mathbb{Z}} V_{j}$ is dense in $L^{2}\left(\mathbb{R}^{+}\right)$, the collection $\left\{\psi_{\ell, j, \lambda}: j \in \mathbb{Z}, \lambda \in \Lambda_{r, N}^{+}, 1 \leq \ell \leq N-1,\right\}$ is an orthonormal basis of $L^{2}\left(\mathbb{R}^{+}\right)$.

\section{Vector-valued nonuniform multiresolution analysis on positive half-line}

We denote the set of all vector-valued functions $\mathbf{f}(x)$ by $L^{2}\left(\mathbb{R}^{+}, \mathbb{C}^{d}\right)$,i.e.

$$
L^{2}\left(\mathbb{R}^{+}, \mathbb{C}^{d}\right)=\left\{\mathrm{f}(x)=\left(f_{1}(x), f_{2}(x), \ldots, f_{d}(x)\right)^{T}: x \in \mathbb{R}^{+}, f_{k}(x) \in L^{2}\left(\mathbb{R}^{+}\right), k=1,2, \ldots, d\right\},
$$

where $T$ denotes the transpose and $\mathbb{C}^{d}$ denotes the $d$-dimensional complex Euclidean space. 
For $\mathbf{f} \in L^{2}\left(\mathbb{R}^{+}, \mathbb{C}^{d}\right),\|\mathbf{f}\|$ denotes the norm of the vector-valued function $\mathbf{f}$ and is defined as:

$$
\|\mathbf{f}\|=\left(\sum_{s=1}^{d} \int_{\mathbb{R}^{+}}\left|f_{s}(x)\right|^{2} d x\right)^{\frac{1}{2}} .
$$

For a vector-valued function $\mathbf{f} \in L^{2}\left(\mathbb{R}^{+}, \mathbb{C}^{d}\right)$ the integration of $\mathbf{f}(x)$ is defined as:

$$
\int_{\mathbb{R}^{+}} \mathbf{f}(x) d x=\left(\int_{\mathbb{R}^{+}} f_{1}(x) d x, \int_{\mathbb{R}^{+}} f_{2}(x) d x, \ldots, \int_{\mathbb{R}^{+}} f_{d}(x) d x\right)^{T} .
$$

The Walsh-Fourier transform of $\mathbf{f}(x)$ is defined by:

$$
\tilde{\mathbf{f}}(\xi)=\int_{\mathbb{R}^{+}} \mathbf{f}(x) \overline{\chi(x, \xi)} d x .
$$

For any two vector-valued functions $\mathbf{f}, \mathbf{g} \in L^{2}\left(\mathbb{R}^{+}, \mathbb{C}^{d}\right),<\mathbf{f}, \mathbf{g}>$ denotes the integration of the matrix product $f(x) \cdot g^{*}(x)$ as:

$$
<\mathbf{f}, \mathbf{g}>=\int_{\mathbb{R}^{+}} \mathbf{f}(x) \mathbf{g}^{*}(x) d x
$$

where $*$ denotes the transpose and the complex conjugate and $\langle\mathbf{f}, \mathbf{g}\rangle$ is matrix valued not scalar valued but satisfied the inner product properties: (i)linearity $<\mathbf{f}, a_{1} g_{1}+a_{2} g_{2}>=a_{1}^{*}<\mathbf{f}, g_{1}>+a_{2}^{*}<\mathbf{f}, g_{2}>$ and (ii) commutativity $<\mathbf{f}, \mathbf{g}>=<\mathbf{g}, \mathbf{f}>^{*}$.

Therefore, the operation $\langle.,$.$\rangle is called an inner product.$

Definition 3.1. Let $N$ be an integer, $N \geq 1$, and $\Lambda_{r, N}^{+}=\{0, r / N\}+\mathbb{Z}^{+}$, where $r$ is an odd integer relatively prime to $N$ with $1 \leq r \leq 2 N-1$. A sequence $\left\{V_{j}: j \in \mathbb{Z}\right\}$ of closed subspaces of $L^{2}\left(\mathbb{R}^{+}, \mathbb{C}^{d}\right)$ is called a vector-valued nonuniform multiresolution analysis (VNUMRA) on positive half-line if the following conditions are satisfied:

(1) $V_{j} \subset V_{j+1} \forall j \in \mathbb{Z}$,

(2) $\overline{\bigcup_{j \in \mathbb{Z}} V_{j}}=L^{2}\left(\mathbb{R}^{+}, \mathbb{C}^{d}\right)$,

(3) $\bigcap_{j \in \mathbb{Z}} V_{j}=\{\mathbf{0}\}$, where $\mathbf{0}$ is the zero vector of $L^{2}\left(\mathbb{R}^{+}, \mathbb{C}^{d}\right)$,

(4) $\Phi(x) \in V_{j}$ if and only if $\Phi(N x) \in V_{j+1}$.

(5) There exists a function $\Phi(x)$ in $V_{0}$ such that $\left\{\Phi(x \ominus \lambda): \lambda \in \Lambda_{r, N}^{+}\right\}$is an orthonormal basis for $V_{0}$.

The vector-valued function $\Phi(x)$ whose existence is asserted in (5) is called a scaling function in $L^{2}\left(\mathbb{R}^{+}, \mathbb{C}^{d}\right)$.

Since $\Phi(x)=\left(\phi_{1}(x), \phi_{2}(x), \ldots, \phi_{d}(x)\right)^{T} \in L^{2}\left(\mathbb{R}^{+}, \mathbb{C}^{d}\right)$, satisfy the following refinement equation

$$
\Phi(x)=\sum_{\lambda \in \Lambda_{r, N}^{+}} H_{\lambda} \Phi(N x \ominus \lambda)
$$

where $\left\{H_{\lambda}\right\}_{\lambda \in \Lambda_{r, N}^{+}}$is $d \times d$ constant matrix sequence that has only finite number of terms.

Define a closed subspace $V_{j} \subset L^{2}\left(\mathbb{R}^{+}, \mathbb{C}^{d}\right)$ by

$$
V_{j}=\operatorname{clos}_{L^{2}\left(\mathbb{R}^{+}, \mathbb{C}^{d}\right)}\left(\operatorname{span}\left\{\Phi\left(N^{j} x \ominus \lambda\right): \lambda \in \Lambda_{r, N}^{+}\right\}\right), \quad j \in \mathbb{Z} .
$$


Given a VNUMRA on positive half-line, we denote $W_{m}$ the orthogonal complement of $V_{m}$ in $V_{m+1}$. Then

$$
L^{2}\left(\mathbb{R}^{+}, \mathbb{C}^{d}\right)=\bigoplus_{m \in \mathbb{Z}} W_{m}
$$

Definition 3.2. A collection of $N-1$ vector-valued functions $\Psi_{k}(t) \in V_{1}, k=1,2, \ldots, N-1$ is called a vectorvalued wavelet system associated with a given VNUMRA on positive half-line if the family of functions $\left\{\Psi_{k}(x \ominus \lambda)\right\}_{k=1,2, \ldots, N-1, \lambda \in \Lambda_{r, N}^{+}}$form an orthonormal basis of $W_{0}$.

Taking the Walsh-Fourier transform on both side of Equation (3.1), we have

$$
\hat{\Phi}(N \eta)=H(\eta) \hat{\Phi}(\eta), \quad \eta \in \mathbb{R}^{+},
$$

where

$$
H(\eta)=\frac{1}{N} \sum_{\lambda \in \Lambda_{r, N}^{+}} H_{\lambda} \overline{\chi(\lambda, \eta)}
$$

and

$$
\chi(\lambda, \eta)=\exp \left(\frac{2 \pi i}{N} \sum_{j=1}^{\infty}\left(\lambda_{j} \xi_{-j}+\lambda_{-j} \xi_{j}\right)\right)
$$

where $\lambda_{j}$ and $\xi_{j}$ are calculated by (2.1).

Since $\Lambda_{r, N}^{+}=\{0, r / N\}+\mathbb{Z}^{+}$, we can write that

$$
H(\eta)=H_{\lambda}^{1}+\overline{\chi\left(\frac{r}{N}, \eta\right)} H_{\lambda}^{2}
$$

where $\left\{H_{\lambda}^{1}\right\}$ and $\left\{H_{\lambda}^{2}\right\}$ are $d \times d$ constant symmetric matrix sequences. Then

$$
\begin{aligned}
\hat{\Phi}(\eta) & =H\left(\frac{\eta}{N}\right) \hat{\Phi}\left(\frac{\eta}{N}\right) \\
& =H\left(\frac{\eta}{N}\right) H\left(\frac{\eta}{N^{2}}\right) H\left(\frac{\eta}{N^{3}}\right) \ldots . . \hat{\Phi}(0) \\
& =\prod_{k=1}^{\infty} H\left(\frac{\eta}{N^{k}}\right) \hat{\Phi}(0)
\end{aligned}
$$

Eq. (3.6) implies that

$$
H(0)=I_{d} \quad \text { or } \quad \sum_{\lambda \in \Lambda_{r, N}^{+}} H_{\lambda}=I_{d}
$$

where $I_{d}$, denotes the identity matrix of order $d \times d$

$$
W_{j}=\operatorname{clos}_{L^{2}\left(\mathbb{R}^{+}, \mathbb{C}^{d}\right)}\left(\operatorname{span}\left\{\Psi_{k}\left(N^{j} x \ominus \lambda\right): \lambda \in \Lambda_{r, N}^{+}, k=1,2, \ldots, N-1\right\}\right), \quad j \in \mathbb{Z} .
$$

Since $\Psi_{k}(x) \in W_{0} \subset V_{1}$, for each $k$, there exists a uniquely supported sequence $\left\{G_{\lambda, k}\right\}_{\lambda \in \Lambda_{r, N}^{+}, k=1,2, \ldots, N-1}$ of $d \times d$ constant matrices such that

$$
\Psi_{k}(x)=\sum_{\lambda \in \Lambda_{r, N}^{+}} G_{\lambda, k} \Phi(N x \ominus \lambda)
$$


On taking Walsh-Fourier transform on both sides of Eq. (3.8), we have

$$
\tilde{\Psi}_{k}(N \eta)=G_{k}(\eta) \tilde{\Phi}(\eta)
$$

where

$$
G_{k}(\eta)=\frac{1}{N} \sum_{\lambda \in \Lambda_{r, N}^{+}} G_{\lambda, k} \overline{\chi(\lambda, \eta)}
$$

and

$$
\chi(\lambda, \eta)=\exp \left(\frac{2 \pi i}{N} \sum_{j=1}^{\infty}\left(\lambda_{j} \xi_{-j}+\lambda_{-j} \xi_{j}\right)\right)
$$

where $\lambda_{j}$ and $\xi_{j}$ are given by (2.1) for a positive integer $N$.

Since $\Lambda_{r, N}^{+}=\{0, r / N\}+\mathbb{Z}^{+}$, we can write that

$$
G_{k}(\eta)=G_{\lambda, k}^{1}+\overline{\chi\left(\frac{r}{N}, \eta\right)} G_{\lambda, k}^{2}, k=0,1, \ldots, N-1,
$$

where $\left\{G_{\lambda, k}^{1}\right\}$ and $\left\{G_{\lambda, k}^{2}\right\}$ are $d \times d$ constant symmetric matrix sequences.

Lemma 3.3. If $\Phi(t) \in L^{2}\left(\mathbb{R}^{+}, \mathbb{C}^{d}\right)$ defined by Eq. (3.1) is an orthogonal vector-valued scaling function then we have

$$
\sum_{m \in \mathbb{Z}^{+}} H_{m} H_{N\left(\lambda \ominus \lambda^{\prime}\right) \oplus m}^{*}=N \delta_{\lambda, \lambda^{\prime}} I_{d}, \quad \forall \lambda, \lambda^{\prime} \in \Lambda_{r, N}^{+}
$$

where $\delta_{\lambda, \lambda^{\prime}}$ denotes the Kronecker's delta.

Proof. Since $\Phi(t) \in L^{2}\left(\mathbb{R}^{+}, \mathbb{C}^{d}\right)$ defined by Eq. (3.1) are orthogonal vector-valued scaling functions, therefore for $\lambda, \lambda^{\prime} \in \Lambda_{r, N}^{+}$, we have

$$
\begin{aligned}
\delta_{\lambda, \lambda^{\prime}} I_{d} & =\int_{\mathbb{R}^{+}} \Phi(x \ominus \lambda) \Phi^{*}\left(x \ominus \lambda^{\prime}\right) d x \\
& =\sum_{u \in \Lambda_{r, N}^{+}} \int_{\mathbb{R}^{+}} H_{u} \Phi(N x \ominus N \lambda \ominus u) \sum_{v \in \Lambda_{r, N}^{+}} \Phi^{*}\left(N x \ominus N \lambda^{\prime} \ominus v\right) H_{v}^{*} d x \\
& =\sum_{u \in \Lambda_{r, N}^{+}} \sum_{v \in \Lambda_{r, N}^{+}} H_{u}\left(\int_{\mathbb{R}^{+}} \Phi(N x \ominus N \lambda \ominus u) \Phi^{*}\left(N x \ominus N \lambda^{\prime} \ominus v\right) d x\right) H_{v}^{*} \\
& =\frac{1}{N} \sum_{u \in \Lambda_{r, N}^{+}} \sum_{v \in \Lambda_{r, N}^{+}} H_{u}\left(\int_{\mathbb{R}^{+}} \Phi(x \ominus N \lambda \ominus u) \Phi^{*}\left(x \ominus N \lambda^{\prime} \ominus v\right) d x\right) H_{v}^{*}
\end{aligned}
$$

Taking $u=m, v=n$, where $m, n \in \mathbb{Z}^{+}$, we have 


$$
\begin{aligned}
\delta_{\lambda, \lambda^{\prime}} I_{d} & =\int_{\mathbb{R}^{+}} \Phi(x \ominus \lambda) \Phi^{*}\left(x \ominus \lambda^{\prime}\right) d x \\
& =\frac{1}{N} \sum_{u \in \Lambda_{r, N}^{+}} \sum_{v \in \Lambda_{r, N}^{+}} H_{u}\left\langle\Phi(x \ominus N \lambda \ominus u), \Phi\left(x \ominus N \lambda^{\prime} \ominus v\right)\right\rangle H_{v}^{*} \\
& =\frac{1}{N} \sum_{m \in \mathbb{Z}^{+}} \sum_{n \in \mathbb{Z}^{+}} H_{m}\left\langle\Phi(x \ominus N \lambda \ominus m), \Phi\left(x \ominus N \lambda^{\prime} \ominus n\right)\right\rangle H_{n}^{*} \\
& =\frac{1}{N} \sum_{m \in \mathbb{Z}^{+}} H_{m} H_{N\left(\lambda \ominus \lambda^{\prime}\right) \oplus m}^{*} .
\end{aligned}
$$

Therefore,

$$
\sum_{m \in \mathbb{Z}^{+}} H_{m} H_{N\left(\lambda \ominus \lambda^{\prime}\right) \oplus m}^{*}=N \delta_{\lambda, \lambda^{\prime}} I_{d}, \forall \lambda, \lambda^{\prime} \in \Lambda_{r, N}^{+} .
$$

Taking $u=\frac{r}{N}+m, v=n$, where $m, n \in \mathbb{Z}^{+}$, we have

$$
\begin{aligned}
\delta_{\lambda, \lambda^{\prime}} I_{d} & =\frac{1}{N} \sum_{u \in \Lambda_{r, N}^{+}} \sum_{v \in \Lambda_{r, N}^{+}} H_{u}\left\langle\Phi(x \ominus N \lambda \ominus u), \Phi\left(x \ominus N \lambda^{\prime} \ominus v\right)\right\rangle H_{v}^{*} \\
& =\frac{1}{N} \sum_{m \in \mathbb{Z}^{+}} \sum_{n \in \mathbb{Z}^{+}} H_{\frac{r}{N}+m}\left\langle\Phi\left(x \ominus N \lambda \ominus\left(\frac{r}{N}+m\right)\right), \Phi\left(x \ominus N \lambda^{\prime} \ominus n\right)\right\rangle H_{n}^{*} \\
& =\frac{1}{N} \sum_{m \in \mathbb{Z}^{+}} H_{m} H_{N\left(\lambda \ominus \lambda^{\prime}\right) \oplus m}^{*} .
\end{aligned}
$$

Therefor in both the cases, we get

$$
\sum_{m \in \mathbb{Z}^{+}} H_{m} H_{N\left(\lambda \ominus \lambda^{\prime}\right) \oplus m}^{*}=N \delta_{\lambda, \lambda^{\prime}} I_{d}, \forall \lambda, \lambda^{\prime} \in \Lambda_{r, N}^{+}
$$

Lemma 3.4. Let $\Psi_{0}=\Phi, G_{0}=H$ and suppose that $\Psi_{k}(t)$, where $k \in \mathcal{D}_{1}=\{1,2, \ldots, N-1\}$ be the vector-valued functions in $V_{1}$. Then the family of functions $\left\{\Psi_{k}(x \ominus \lambda)\right\}_{\lambda \in \Lambda_{r, N}^{+}, k=0,1,2, \ldots, N-1}$ will forms an orthonormal system for $V_{1}$ if and only if for $k, l \in\{0,1,2, \ldots, N-1\}$

$$
\sum_{p=0}^{N-1} G_{k}\left(\frac{\xi+p}{N}\right) G_{l}^{*}\left(\frac{\xi+p}{N}\right)=\delta_{k, l} I_{d} .
$$

Proof. First, we will prove the necessary condition.

By orthonormality of $\Psi_{k}(x) \in L^{2}\left(\mathbb{R}^{+}, \mathbb{C}^{d}\right), k=0,1,2, \ldots, N-1$, we have

$$
\left\langle\Psi_{k}(x), \Psi_{l}^{*}(x \ominus \lambda)\right\rangle=\int_{\mathbb{R}^{+}} \Psi_{k}(x) \Psi_{l}^{*}(x \ominus \lambda) d t=\delta_{k, l} \delta_{0, \lambda} I_{d}
$$

where $\lambda \in \Lambda_{r, N}^{+}$and $k, l \in\{0,1,2, \ldots, N-1\}$.

Equivalently, in the frequency domain, we have

$$
\delta_{k, l} \delta_{0, \lambda} I_{d}=\int_{\mathbb{R}^{+}} \tilde{\Psi}_{k}(\xi) \tilde{\Psi}_{l}^{*}(\xi) \overline{\chi(\lambda, \xi)} d \xi .
$$


Define

$$
F_{k, l}(\xi)=\sum_{j \in \mathbb{Z}^{+}} \tilde{\Psi}_{k}(\xi+N j) \tilde{\Psi}_{l}^{*}(\xi+N j) .
$$

On taking $\lambda=n$, where $n \in \mathbb{Z}^{+}, 0 \leq k, l \leq N-1$, we have

$$
\begin{aligned}
\delta_{k, l} \delta_{0, n} I_{d} & =\int_{\mathbb{R}^{+}} \tilde{\Psi}_{k}(\xi) \tilde{\Psi}_{l}^{*}(\xi) \overline{\chi(n, \xi)} d \xi \\
& =\int_{[0, N)} \sum_{j \in \mathbb{Z}^{+}} \tilde{\Psi}_{k}(\xi+N j) \tilde{\Psi}_{l}^{*}(\xi+N j) \overline{\chi(n, \xi)} d \xi \\
& =\int_{[0, N)} F_{k, l}(\xi) \overline{\chi(n, \xi)} d \xi \\
& =\int_{[0,1)}\left[\sum_{p=0}^{N-1} F_{k, l}(\xi+p)\right] \overline{\chi(n, \xi)} d \xi .
\end{aligned}
$$

Therefore, we obtain

$$
\sum_{p=0}^{N-1} F_{k, l}(\xi+p)=\delta_{k, l} I_{d}
$$

On taking $\lambda=\frac{r}{N}+n$, where $n \in \mathbb{Z}^{+}$, we obtain

$$
\begin{aligned}
0 & =\int_{\mathbb{R}^{+}} \tilde{\Psi}_{k}(\xi) \tilde{\Psi}_{l}^{*}(\xi) \overline{\chi\left(\frac{r}{N}+n, \xi\right)} d \xi \\
& =\int_{[0, N)} \sum_{j \in \mathbb{Z}^{+}} \tilde{\Psi}_{k}(\xi+N j) \tilde{\Psi}_{l}^{*}(\xi+N j) \overline{\chi(n, \xi)} \overline{\chi\left(\frac{r}{N}, \xi\right)} d \xi \\
& =\int_{[0, N)} F_{k, l}(\xi) \overline{\chi(n, \xi)} \overline{\chi\left(\frac{r}{N}, \xi\right)} d \xi \\
& =\int_{[0,1)}\left[\sum_{p=0}^{N-1} \overline{\chi\left(\frac{r}{N}, p\right)} F_{k, l}(\xi+p)\right] \overline{\chi(n, \xi)} \overline{\chi\left(\frac{r}{N}, \xi\right)} d \xi
\end{aligned}
$$

We conclude that

$$
\sum_{p=0}^{N-1} \overline{\chi\left(\frac{r}{N}, p\right)} F_{k, l}(\xi+p)=0
$$

i.e.

$$
\sum_{p=0}^{N-1} \alpha^{p} F_{k, l}(\xi+p)=0,
$$

where $\alpha=\overline{\chi\left(\frac{r}{N}, 1\right)}$, since $\overline{\chi\left(\frac{r}{N}, p\right)}=\overline{\left[\chi\left(\frac{r}{N}, 1\right)\right]^{p}}$ for $p=0,1,2, \ldots, N-1$.

Also, we have

$$
\sum_{p=0}^{N-1} F_{k, l}(\xi+p)=\sum_{p \in \mathbb{Z}^{+}} \tilde{\Psi}_{k}(\xi+p) \tilde{\Psi}_{l}^{*}(\xi+p) .
$$


Therefore, Eq.(3.14) reduces to

$$
\sum_{p \in \mathbb{Z}^{+}} \tilde{\Psi}_{k}(\xi+p) \tilde{\Psi}_{l}^{*}(\xi+p)=\delta_{k, l} I_{d}
$$

Also,

$$
\begin{aligned}
& F_{k, l}(N \xi)=\sum_{j \in \mathbb{Z}^{+}} \tilde{\Psi}_{k}(N \xi+N j) \tilde{\Psi}_{l}^{*}(N \xi+N j) \\
& =\sum_{j \in \mathbb{Z}^{+}} G_{k}(\xi+j) \tilde{\Phi}(\xi+j) \tilde{\Phi}^{*}(\xi+j) G_{l}^{*}(\xi+j) \\
& =\sum_{j=n N} G_{k}(\xi+n N) \tilde{\Phi}(\xi+n N) \tilde{\Phi}^{*}(\xi+n N) G_{l}^{*}(\xi+n N) \\
& +\sum_{j=n N+1} G_{k}(\xi+n N+1) \tilde{\Phi}(\xi+n N+1) \tilde{\Phi}^{*}(\xi+n N+1) \\
& \times G_{l}^{*}(\xi+n N+1)+\sum_{j=n N+2} G_{k}(\xi+n N+2) \tilde{\Phi}(\xi+n N+2) \\
& \times \tilde{\Phi}^{*}(\xi+n N+2) G_{l}^{*}(\xi+n N+2)+\ldots+ \\
& \begin{array}{l}
\sum_{\substack{j=n N+(N-1) \\
\times \tilde{\Phi}^{*}(\xi+n N+(N-1)) G_{l}^{*}(\xi+n N+(N-1))}} G_{k}(\xi+n N+(N-1)) \tilde{\Phi}(\xi+n N+(N-1)) \\
\end{array} \\
& =G_{k}(\xi)\left[\sum_{j=n N} \tilde{\Phi}(\xi+n N) \tilde{\Phi}^{*}(\xi+n N)\right] G_{l}^{*}(\xi)+G_{k}(\xi+1) \\
& \times\left[\sum_{j=n N+1} \tilde{\Phi}(\xi+n N+1) \tilde{\Phi}^{*}(\xi+n N+1)\right] G_{l}^{*}(\xi+1) \\
& +G_{k}(\xi+2)\left[\sum_{j=n N+2} \tilde{\Phi}(\xi+n N+2) \tilde{\Phi}^{*}(\xi+n N+2)\right] \\
& \times G_{l}^{*}(\xi+2)+\ldots+G_{k}(\xi+(N-1)) \\
& \times\left[\sum_{\substack{j=n N+(N-1) \\
\times G_{l}^{*}(\xi+(N-1))}} \tilde{\Phi}(\xi+n N+(N-1)) \tilde{\Phi}^{*}(\xi+n N+(N-1))\right] \\
& =G_{k}(\xi) G_{l}^{*}(\xi)+G_{k}(\xi+1) G_{l}^{*}(\xi+1)+G_{k}(\xi+2) G_{l}^{*}(\xi+2)+\ldots \\
& +G_{k}(\xi+(N-1)) G_{l}^{*}(\xi+(N-1)) \\
& =\sum_{j=0}^{N-1} G_{k}(\xi+j) G_{l}^{*}(\xi+j) .
\end{aligned}
$$

Therefore, we have

$$
\sum_{p \in \mathbb{Z}^{+}} \tilde{\Psi}_{k}(\xi+p) \tilde{\Psi}_{l}^{*}(\xi+p)=\sum_{p=0}^{N-1} G_{k}\left(\frac{\xi+p}{N}\right) G_{l}^{*}\left(\frac{\xi+p}{N}\right) .
$$

By using (3.16), we conclude that 


$$
\sum_{p=0}^{N-1} G_{k}\left(\frac{\xi+p}{N}\right) G_{l}^{*}\left(\frac{\xi+p}{N}\right)=\delta_{k, l} I_{d}
$$

i.e.

$$
\begin{gathered}
G_{k}\left(\frac{\xi}{N}\right) G_{l}^{*}\left(\frac{\xi}{N}\right)+G_{k}\left(\frac{\xi+1}{N}\right) G_{l}^{*}\left(\frac{\xi+1}{N}\right)+G_{k}\left(\frac{\xi+2}{N}\right) \\
G_{l}^{*}\left(\frac{\xi+2}{N}\right)+\ldots+G_{k}\left(\frac{\xi+N-1}{N}\right) G_{l}^{*}\left(\frac{\xi+N-1}{N}\right)=\delta_{k, l} I_{d} .
\end{gathered}
$$

Now, we will prove the sufficiency.

By Eq.(3.9), we have

$$
\begin{aligned}
\sum_{j \in \mathbb{Z}^{+}} & \tilde{\Psi}_{k}(\xi+j) \tilde{\Psi}_{l}^{*}(\xi+j) \\
= & \sum_{j \in \mathbb{Z}^{+}} G_{k}\left(\frac{\xi+j}{N}\right) \tilde{\Phi}\left(\frac{\xi+j}{N}\right) \tilde{\Phi}^{*}\left(\frac{\xi+j}{N}\right) G_{l}^{*}\left(\frac{\xi+j}{N}\right) \\
= & G_{k}\left(\frac{\xi}{N}+n\right)\left[\sum_{j=n N} \tilde{\Phi}\left(\frac{\xi}{N}+n\right) \tilde{\Phi}^{*}\left(\frac{\xi}{N}+n\right)\right] \\
& \times G_{l}^{*}\left(\frac{\xi}{N}+n\right)+G_{k}\left(\frac{\xi}{N}+n+\frac{1}{N}\right) \\
& \times\left[\sum_{j=n N+1} \tilde{\Phi}\left(\frac{\xi}{N}+n+\frac{1}{N}\right) \tilde{\Phi}^{*}\left(\frac{\xi}{N}+n+\frac{1}{N}\right)\right] G_{l}^{*}\left(\frac{\xi}{N}+n+\frac{1}{N}\right) \\
& +\ldots+G_{k}\left(\frac{\xi}{N}+n+\frac{N-1}{N}\right)\left[\sum_{j=n N+(N-1)}\left(\frac{\xi}{N}+n+\frac{N-1}{N}\right)\right. \\
& \left.\times \tilde{\Phi}^{*}\left(\frac{\xi}{N}+n+\frac{N-1}{N}\right)\right] G_{l}^{*}\left(\frac{\xi}{N}+n+\frac{\tilde{\Phi}}{N}\right) \\
= & G_{k}\left(\frac{\xi}{N}\right) G_{l}^{*}\left(\frac{\xi}{N}\right)+G_{k}\left(\frac{\xi+1}{N}\right) G_{l}^{*}\left(\frac{\xi+1}{N}\right)+G_{k}\left(\frac{\xi+2}{N}\right) \\
& \times G_{l}^{*}\left(\frac{\xi+2}{N}\right)+\ldots+G_{k}\left(\frac{\xi+N-1}{N}\right) G_{l}^{*}\left(\frac{\xi+N-1}{N}\right) \\
= & \delta_{k, l} I_{d} .
\end{aligned}
$$

It proves the orthonormality of the system $\left\{\Psi_{k}(x \ominus \lambda): \lambda \in \Lambda_{r, N}^{+}, k=0,1,2, \ldots, N-1\right\}$.

Now, we have the following result on the existence of a vector-valued wavelet function

Theorem 3.5. Suppose $\left\{\Psi_{k}(x \ominus \lambda)\right\}_{\lambda \in \Lambda_{r, N}^{+}, k=0,1,2, \ldots, N-1}$ is an orthonormal system in $V_{1}$. Then this system is complete in $W_{0} \equiv V_{1} \ominus V_{0}$.

Proof. Since the system $\left\{\Psi_{k}(x \ominus \lambda)\right\}_{\lambda \in \Lambda_{r, N}^{+}, k=0,1,2, \ldots, N-1}$ is an orthonormal system in $V_{1}$. By Lemma 3.4, we have

$$
G_{k}\left(\frac{\xi}{N}\right) G_{l}^{*}\left(\frac{\xi}{N}\right)+G_{k}\left(\frac{\xi+1}{N}\right) G_{l}^{*}\left(\frac{\xi+1}{N}\right)+G_{k}\left(\frac{\xi+2}{N}\right)
$$




$$
G_{l}^{*}\left(\frac{\xi+2}{N}\right)+\ldots+G_{k}\left(\frac{\xi+(N-1)}{N}\right) G_{l}^{*}\left(\frac{\xi+(N-1)}{N}\right)=\delta_{k, l} I_{d} .
$$

We will now prove its completeness.

For $\mathbf{f}_{k} \in W_{0}$, there exists constant matrices $\left\{P_{\lambda, k}\right\}$ such that

$$
\mathbf{f}_{k}(t)=\sum_{\lambda \in \Lambda_{r, N}^{+}} P_{\lambda, k} \Phi(N t \ominus \lambda), k=0,1,2, \ldots, N-1 .
$$

Thus

$$
\tilde{\mathbf{f}}_{k}(\xi)=P_{k}\left(\frac{\xi}{N}\right) \tilde{\Phi}\left(\frac{\xi}{N}\right)
$$

where

$$
P_{k} \xi=\frac{1}{N} \sum_{\lambda \in \Lambda_{r, N}^{+}} P_{\lambda, k} \overline{\chi(\lambda, \xi)}
$$

On the other hand $\mathbf{f}_{k} \notin V_{0}$ and $\mathbf{f}_{k} \in W_{0}$ implies

$$
\int_{\mathbb{R}^{+}} \mathbf{f}_{k}(t) \Phi^{*}(t \ominus \lambda) d t=0, \quad \lambda \in \Lambda_{r, N}^{+} .
$$

This condition is equivalent to

$$
\sum_{j \in \mathbb{Z}^{+}} \tilde{\mathbf{f}}_{k}(\xi+j) \tilde{\Phi}^{*}(\xi+j)=0, \quad \xi \in \mathbb{R}^{+} .
$$

Therefore, from Eqs. (3.3) and (3.17), we have

$$
\sum_{j \in \mathbb{Z}^{+}} P_{k}\left(\frac{\xi+j}{N}\right) \tilde{\Phi}\left(\frac{\xi+j}{N}\right) \tilde{\Phi}^{*}\left(\frac{\xi+j}{N}\right) H^{*}\left(\frac{\xi+j}{N}\right)=0, \quad \xi \in \mathbb{R}^{+} .
$$

As similar to the identity (3.13) in Lemma 3.4, we have

$$
\begin{gathered}
P_{k}\left(\frac{\xi}{N}\right) H^{*}\left(\frac{\xi}{N}\right)+P_{k}\left(\frac{\xi+1}{N}\right) H^{*}\left(\frac{\xi+1}{N}\right)+\ldots+ \\
P_{k}\left(\frac{\xi+(N-1)}{N}\right) H^{*}\left(\frac{\xi+(N-1)}{N}\right)=0 .
\end{gathered}
$$

Let

$$
\begin{aligned}
P_{k^{\prime}}\left(\frac{\xi}{N}\right) & =\left(P_{k}\left(\frac{\xi}{N}\right), P_{k}\left(\frac{\xi+1}{N}\right), \ldots, P_{k}\left(\frac{\xi+(N-1)}{N}\right)\right)^{*}, \\
H^{\prime}\left(\frac{\xi}{N}\right) & =\left(H\left(\frac{\xi}{N}\right), H\left(\frac{\xi+1}{N}\right), \ldots, H\left(\frac{\xi+(N-1)}{N}\right)\right)^{*}, \\
G_{k^{\prime}}\left(\frac{\xi}{N}\right) & =\left(G_{k}\left(\frac{\xi}{N}\right), G_{k}\left(\frac{\xi+1}{N}\right), \ldots, G_{k}\left(\frac{\xi+(N-1)}{N}\right)\right)^{*} .
\end{aligned}
$$


Then, the identity (3.13) implies that for any $\xi \in \mathbb{R}^{+}$, the column vectors in the $N d \times d$ matrix $H^{\prime}(\xi)$ and the column vectors in the $N d \times d$ matrix $G_{k}^{\prime}(\xi)$ are orthogonal for $l=0,1,2, \ldots, N-1$ and these vectors form orthogonal basis of $N d$-dimensional complex Eucledian space $\mathbb{C}^{N d}$.

The identity (3.18) implies that the column vectors in $N d \times d$ matrix $P_{k}^{\prime}(\xi)$ and the column vectors in $N d \times d$ matrix $H^{\prime}(\xi)$ are orthogonal. Thus $\exists$ an $d \times d$ matrix $Q_{k}(\xi)$ such that

$$
P_{k}(\xi)=Q_{k}(\xi) G_{k}(\xi), \quad \xi \in \mathbb{R}^{+}, \quad k=0,1,2, \ldots, N-1 .
$$

Therefore, from Eqs. (3.9) and (3.17), we have

$$
\begin{aligned}
\hat{\mathbf{f}}_{k}(\xi) & =P_{k}\left(\frac{\xi}{N}\right) \tilde{\Phi}\left(\frac{\xi}{N}\right) \\
& =P_{k}\left(\frac{\xi}{N}\right) G_{k}\left(\frac{\xi}{N}\right) \tilde{\Phi}\left(\frac{\xi}{N}\right) \\
& =Q_{k}\left(\frac{\xi}{N}\right) \tilde{\Psi}_{k}(\xi) .
\end{aligned}
$$

By using the orthonormality of $\left\{\Psi_{k}(x \ominus \lambda): \lambda \in \Lambda_{r, N}^{+}\right\}$, we have

$$
\int_{\mathbb{R}^{+}} \tilde{\mathbf{f}}_{k}(N \xi) \tilde{\mathbf{f}}_{k}^{*}(N \xi) d \xi=\int_{\mathbb{R}^{+}} Q_{k}(\xi) \tilde{\Psi}_{k}(N \xi) \tilde{\Psi}_{k}^{*}(N \xi) Q_{k}^{*}(\xi) d \xi
$$

Therefore, we have

$$
\int_{\mathbb{R}^{+}} \tilde{\mathbf{f}}_{k}(N \xi) \tilde{\mathbf{f}}_{k}^{*}(N \xi) d \xi=\int_{0}^{1} Q_{k}(\xi) Q_{k}^{*}(\xi) d \xi
$$

This proves that $P_{k}(\xi)$ has Fourier series expansion and let the constant $d \times d$ matrices $\left\{A_{\lambda, k}\right\}_{\lambda \in \Lambda_{r, N}^{+}, k=0,1,2, \ldots, N-1}$ be its Fourier coefficients. Therefore,

$$
\mathbf{f}_{k}(x)=\sum_{\lambda \in \Lambda_{r, N}^{+}} A_{\lambda, k} \Psi_{k}(x \ominus \lambda) .
$$

This proves the completeness of $\left\{\Psi_{k}(x \ominus \lambda)\right\}_{\lambda \in \Lambda_{r, N}^{+}, k=0,1,2, \ldots, N-1}$ in $W_{0}$.

If $\Psi_{0}, \Psi_{1}, \Psi_{2}, \ldots, \Psi_{N-1} \in V_{1}$ are as in Theorem 3.5, one can obtain from them as orthonormal basis for $L^{2}\left(\mathbb{R}^{+}, \mathbb{C}^{d}\right)$ by procedure for construction of wavelet from given MRA. It can be easily checked that for every $j \in \mathbb{Z}$ the collection

$$
\left\{N^{\frac{m}{2}} \Psi_{k}\left(N^{m} t \ominus \lambda\right)\right\}_{\lambda \in \Lambda_{r, N}^{+}, k=0,1,2, \ldots, N-1},
$$

is a complete orthogonal system for $V_{m+1}$.

For every $m \in \mathbb{Z}$, define $W_{m}$ the orthogonal complement of $V_{m}$ in $V_{m+1}$. Then we have

$$
V_{m+1}=V_{m} \oplus W_{m} \text { and } W_{k} \perp W_{l} \text { if } k \neq l
$$

and

$$
L^{2}\left(\mathbb{R}^{+}, \mathbb{C}^{d}\right)=\bigoplus_{m \in \mathbb{Z}} W_{m}
$$

a decomposition of $L^{2}\left(\mathbb{R}^{+}, \mathbb{C}^{d}\right)$ into mutually orthogonal subspaces. 
The collection $\left\{N^{\frac{m}{2}} \Psi_{k}\left(N^{m} t \ominus \lambda\right)\right\}_{\lambda \in \Lambda_{r, N}^{+}, m \in \mathbb{Z} k=0,1,2, \ldots, N-1}$ forms a complete orthonormal system for $L^{2}\left(\mathbb{R}^{+}, \mathbb{C}^{d}\right)$.

\section{Conclusion}

In this study, we introduced a new concept called vector-valued nonuniform multiresolution analysis on positive half-line related to Walsh functions where associated subspace $V_{0}$ of $L^{2}\left(\mathbb{R}^{+}, \mathbb{C}^{d}\right)$ has, an orthonormal basis, a collection of translates of vector-valued function $\Phi$ of the form $\{\Phi(x \ominus \lambda)\}_{\lambda \in \Lambda_{r, N}^{+}}$. We Also obtained the necessary and sufficient condition for the existence of associated wavelets. Our results will be mostly used by that part of mathematical society who works in wavelet analysis and their applications.

\section{Acknowledgement}

We are deeply indebted to the referee for his/her valuable suggestions which greatly improved the presentation of this paper.

\section{REFERENCES}

1. Abdullah, Vector-valued multiresolution analysis on local fields, Analysis, Analysis, Vol. 34, no. 4, pp. 415-428, 2014.

2. K. Ahmad, and Abdullah, Wavelet Packets and Their Statistical Applications, Springer, 2018.

3. Q. Chen, and Z. Cheng, A study on compactly supported orthogonal vector-valued wavelets and wavelet packets, Chaos, Solitons and Fractals, Vol, 31, pp. 1024-1034, 2007.

4. I. Daubechies, Ten Lectures on Wavelets, CBMS-NSF Regional Conference in Applied Mathematics, SIAM, Philadelphia, 1992.

5. Y. A. Farkov, Orthogonal p-wavelets on $\mathbb{R}^{+}$, in Proceedings of International Conference Wavelets and Splines, St. Petersberg State University, St. Petersberg, pp. 4-26, 2005.

6. Y. A. Farkov, A. Y. Maksimov and S. A. Stroganov, On biorthogonal wavelets related to the Walsh functions, Int. J. Wavelets, Multiresolut. Inf. Process., Vol. 9, no. 3, pp. 485-499, 2011.

7. J.-P. Gabardo and M. Nashed, Nonuniform multiresolution analyses and spectral pairs, J. Funct. Anal., Vol. 158, pp. 209-241, 1998.

8. J.-P. Gabardo and M. Nashed, An analogue of Cohen's condition for nonuniform multiresolution analyses, in: A. Aldroubi, E. Lin (Eds.), Wavelets, Multiwavelets and Their Applications, in: Cont. Math., 216, Amer. Math. Soc., Providence, RI, pp. 41-61, 1998.

9. J.-P. Gabardo, and X. Yu,. Wavelets associated with nonuniform multiresolution analyses and one-dimensional spectral pairs, J. Math. Anal. Appl., Vol. 323, pp. 798-817, 2006.

10. G. Gripenberg, A necessary and sufficient condition for the existence of a father wavelet, Stud. Math. 114, pp. 207-226, 1995.

11. E. Hernández and G. Weiss, A First Course on Wavelets, CRC Press, New York, 1996.

12. W.C. Lang, Orthogonal wavelets on the Cantor dyadic group, SIAM J. Math. Anal., Vol. 27, pp. 305-312, 1996.

13. W.C. Lang, Fractal multiwavelets related to the Cantor dyadic group, Int. J. Math. Sci., Vol. 21, pp. 307-314, 1998.

14. W.C. Lang, Wavelet analysis on the Cantor dyadic group, Houston J. Math., Vol. 24, pp. 533-544, 1998.

15. S. G. Mallat, Multiresolution approximations and wavelet orthonormal bases of $L^{2}(\mathbb{R})$, Trans. Amer. Math. Soc., Vol. 315 , pp. $69-$ 87, 1989.

16. Meenakshi, P. Manchanda and A. H. Siddiqi, Wavelets associated with vector-valued nonuniform multiresolution analysis, Applicable Anal., Vol. 93, no. 1, pp. 84-104, 2014.

17. Meenakshi, P. Manchanda and A. H. Siddiqi, Wavelets associated with Nonuniform multiresolution analysis on positive half line, Int. J. Wavelets, Multiresolut. Inf. Process., Vol. 10, no. 2, 2011.

18. V. Yu Protasov and Yu A. Farkov, Dyadic wavelets and refinable function on a half-line, Sbornik: Mathematics, Vol. 197, no. 10, pp. 1529-1558, 2006.

19. F. A. Shah, Construction of wavelets packet on p-adic field, Int. J. Wavelets, Multiresolut. Inf. Process., Vol. 7, no. 5, pp. 553-565, 2009.

20. F. A. Shah, Gabor frames on a half-line, J. contemp. Math. Anal., Vol. 47, no. 5, pp. 251-260, 2012.

21. F. A. Shah, Tight wavelet frames generated by the walsh polynomials, Int. J. Wavelets Multiresolut. Inf. Process., Vol. 11, no. 6, 1350042 (15 pages), 2013.

22. F A Shah and L. Debnath, Dyadic wavelet frames on a half-line using the Walsh-Fourier transform, Integ. Trans. Special Funct., Vol. 22, no. 7, pp. 477-486, 2011.

23. L. Sun and Z. Cheng, Construction of a class of compactly supported orthogonal vector-valued wavelets, Chaos, Solitons and Fractals, Vol. 34, pp. 253-261, 2007.

24. X.G. Xia and B.W. Suter, Vector-valued wavelets and vector filter banks, IEEE Transactions on Signal Processing, Vol. 44, no. 3 , pp. 508-518, 1996 
25. X. Yu and J-P Gabardo, Nonuniform wavelets and wavelet sets related to one-dimensional spectral pairs, J. Approx. Theo., Vol. 145, pp. 133-139, 2007.

26. G. Zhou and J. Huang, Adaptive wavelet tight frame construction for accelerating MRI reconstruction, Stat., Optim. Inf. Comput., Vol. 5, pp. 200-211, 2017. 